\title{
Can Multiple Subchannels Improve the Delay Performance of RTS/CTS-based MAC Schemes?
}

\author{
Jing Deng, Member, IEEE, Yunghsiang S. Han, Member, IEEE, and Sanjeev R. Kulkarni, Fellow, IEEE
}

\begin{abstract}
We analyze the delay performance of RTS/CTSbased (Request-To-Send/Clear-To-Send) multi-channel MAC (Medium Access Control) schemes for wireless networks. These schemes usually employ multiple data subchannels for data transmission and one control subchannel to send the RTS/CTS dialogue for channel reservation. Through theoretical analysis and simulations, we show that, in fully-connected networks, such multi-channel MAC schemes suffer longer delays than the corresponding single channel MAC scheme, that puts the RTS/CTS dialogue on the same channel as data packet transmissions. This conclusion holds even when data packets have different priorities and higher priority traffic is sent ahead of lower priority traffic.
\end{abstract}

Index Terms - medium access control (MAC); multiple channels; ALOHA; contention resolution; RTS/CTS dialogue; delay performance

\section{INTRODUCTION}

In wireless communication networks, Medium Access Control (MAC) schemes are used to manage all nodes' access to the shared wireless medium. Due to the randomness of packet arrivals and local competition, it is difficult to completely eliminate packet collisions.

Since data packet collisions are costly, researchers proposed to use the RTS/CTS (Request-To-Send/Clear-To-Send) dialogue to reserve the right to channel usage. In the RTS/CTS technique, a ready node sends an RTS request to the intended receiver. When the RTS packet is heard and the channel is available, the intended receiver replies with a CTS packet, notifying all neighboring nodes of the successful channel reservation. Data packet transmission then follows. With the use of the RTS/CTS dialogue, it is less likely that data packets will suffer collisions.

One class of such RTS/CTS-based MAC schemes separate the shared wireless medium into several subchannels, e.g., [1] and more references can be found in [2]. In these schemes, the RTS/CTS dialogues are sent on one subchannel to reserve the right to use the multiple data subchannels. With such an arrangement, data packets will never collide with control

Manuscript received November 2006; revised January 2008 and September 2008; accepted December 2008. The editor coordinating the review of this paper and approving it for publication is D. Gesbert. This work was supported in part by the National Science Council of Taiwan, R.O.C., under grants NSC 90-2213-E-260-007 and NSC 91-2213-E-260-021. Deng's work was partially supported by his visit to Princeton University in Fall 2005.

J. Deng is with the Dept. of Computer Science, Univ. of North Carolina at Greensboro (E-mail: jing.deng@uncg.edu).

Y. S. Han is with Graduate Institute of Communication Engineering, National Taipei Univ., Taiwan, R.O.C. (E-mail: yshan@mail.ntpu.edu.tw). Corresponding author.

S. R. Kulkarni is with the Dept. of Electrical Engineering, Princeton Univ. (E-mail: kulkarni@princeton.edu). packets as they are sent on different subchannels. Since all data packet transmissions are preceded by RTS/CTS dialogues on the control subchannel, it is rather unlikely that data packet collisions will occur. In order to investigate such multi-channel MAC schemes as a class of schemes, we have ignored some of the features proposed in the original techniques, e.g., power control [1].

In [2], [3], the throughput performance of such schemes have been investigated. It was found that the single channel MAC scheme outperforms the multi-channel MAC schemes in throughput in most scenarios with fully connected networks. However, the delay performance of such multi-channel MAC schemes is still unclear. In this work, we focus on the delay performance comparison of these MAC schemes.

There are several published work related to our work. Marsan and Neri [4] used simulations to study the performance of multi-channel CSMA/CD (Carrier Sense Multiple Access/Collision Detection) schemes on LANs (Local Area Networks). It was found that multi-channel MAC schemes may improve the delay performance. Yue and Matsumoto investigated the throughput and delay performance of integrated voice and data transmission in slotted ALOHA systems that use multiple channels [5]. So and Vaidya took advantage of the available multiple channels and used the ATIM (Ad-hoc Traffic Indication Message) window in their Multi-channel MAC (MMAC) scheme [6]. Xu et al. suggested using multiple transceivers on a single node [7]. Such a scheme becomes more affordable with lowering cost of additional hardware.

Han et al. [2] categorized different MAC schemes into MAC- 1 and MAC- $m D$ schemes. The MAC-1 scheme sends the RTS/CTS control packets and the data packets on the same channel. The MAC- $m D$ schemes separate the single channel into $m$ data subchannels and one control subchannel. The RTS/CTS control packets are sent on the control subchannel. Data packets are sent on the data subchannels. More details on the MAC- $m D$ schemes can be found in [2]. ${ }^{1}$ An example and comparisons between MAC-1 and MAC-2D are illustrated in Fig. 1.

In this work, we focus on the delay performance of such MAC- $m D$ schemes and their comparison to the MAC1 scheme. In particular, we try to answer the following questions: how does the delay performance of multi-channel MAC schemes compare with that of the MAC-1 scheme? When data packets have different priorities, will the MAC- $m D$

\footnotetext{
${ }^{1}$ Please note that we did not propose the MAC-mD scheme. Instead, we summarized the main features of several multi-channel MAC schemes into MAC- $m D$ scheme so that we could compare its performance with the corresponding MAC-1 schemes.
} 
schemes outperform the MAC-1 scheme in delay performance of the high priority packets? The second question is interesting because, intuitively, it may be beneficial to have multiple data subchannels to serve prioritized traffic.

Through theoretical analysis and simulations, we show that, in fully-connected networks, these multi-channel MAC schemes suffer longer delays than the corresponding single channel MAC scheme, that puts the RTS/CTS dialogue on the same channel as data packet transmissions. This conclusion holds even when there are prioritized traffic and data packets are sent based on their priorities.

\section{DELAY ANALYSis}

\section{A. Assumptions and Notations}

We normalize time so that the time to transmit a control packet (either RTS or CTS) is always 1 [time-unit]. ${ }^{2}$ We assume that pure ALOHA contention resolution is used. The overall traffic generated by active nodes (including retransmissions) is Poisson with aggregate arrival rate of $G$ [packets/time-unit]. We further assume that the packet processing and the radio propagation delays are negligible. The total available channel bandwidth is fixed. The wireless communication networks that we study are assumed to be fullyconnected; i.e., every node is in the transmission range of all other nodes in the network. Every reservation and every transmission can be overheard by all nodes in the network. ${ }^{3}$

We establish the following notation:

- $m$ : the number of data subchannels in the MAC- $m D$ scheme;

- $q$ : the number of extra successful channel reservations that are allowed when all $m$ data subchannels are in use;

- $L_{c}, L_{d}$ : the length (in bits) of a control (RTS/CTS) packet and that of a data packet, respectively;

- $k$ : the ratio of data packet size to the control packet size; i.e., $k=\frac{L_{d}}{L_{c}}$;

- $R, R_{c}$, and $R_{d}$ : the data rate (in bits-per-second) of the entire shared channel, the control subchannel, and the data subchannel, respectively; i.e., $R=R_{c}+m R_{d}$;

- $r$ : the ratio of the data rate of the control subchannel to the data rate of the data subchannel in the MAC- $m D$ scheme; i.e., $r=\frac{R_{c}}{R_{d}}$;

- $\delta=1 / \mu$ : data packet transmission time (in time-units) in the MAC- $m D$ scheme; i.e., $\delta=\frac{L_{d} / R_{d}}{L_{c} / R_{c}}=k r$.

Note that our multi-channel model is effectively the same as those used in [8], [9] for fair of comparison between MAC- $m D$ and MAC-1 schemes. Our model is different to those used in [6], [7], which assumes that each individual channel supports the same data rate.

\section{B. Queue Analysis}

We use the $M / M / m / m+q$ model to analyze the MAC- $m D$ schemes. The service time is assumed to be exponentially

\footnotetext{
${ }^{2}$ The use of the RTS/CTS dialogue in a MAC scheme is two-fold: data packet transmission reservation and solving the hidden/exposed terminal problems which appear in multi-hop networks. We focus on its first usage in this work.

${ }^{3}$ The performance study of MAC schemes in multi-hop networks would be very interesting but out of the scope of this work.
}

distributed with mean of $\delta=1 / \mu$. We will discuss the difference between this model and the behavior of the MAC- $m D$ schemes with fixed data packet length in Section III.

Let $\lambda$ be the average completion rate of successful RTS/CTS dialogues in a time-unit on the control subchannel. It can be calculated as ( [2]):

$$
\lambda=\frac{G e^{-2 G}}{1+G e^{-2 G}},
$$

where $G$ is the actual traffic rate going into the ALOHA system (RTS/CTS contention resolution process) on the control subchannel in each unit-time and $G e^{-2 G}$ is the form of ALOHA throughput corresponding to Poisson arrival rate of $G$.

For the $M / M / m / m+q$ model, the steady-state probabilities of $n$ customers in the queue are given as:

$$
\pi_{n}=\left\{\begin{array}{ll}
\pi_{0} \frac{(m \rho)^{n}}{n !}, & n \leq m \\
\pi_{0} \frac{m^{m} \rho^{n}}{m !}, & m<n \leq m+q
\end{array},\right.
$$

where $\rho=\frac{\lambda}{m \mu}$ is the utilization factor and $\pi_{i}$ represents steady state probability of $i$ customers in the queue.

Using the condition $\sum_{n=0}^{m+q} \pi_{n}=1$, we calculate $\pi_{0}$ to be:

$$
\pi_{0}=\frac{1}{\sum_{n=0}^{m} \frac{(m \rho)^{n}}{n !}+\sum_{n=m+1}^{m+q} \frac{(m \rho)^{n}}{m !} \frac{1}{m^{n-m}}} .
$$

\section{Arrivals of Competing Packets}

In the MAC- $m D$ scheme, when the queue is full, all the winners of the competition are forced to join the competition again as new arrivals [2]. Based on the assumption that $q$ successful users may stay in the distributed queue, the probability of a successful RTS/CTS dialogue being dropped in a MAC- $m D$ scheme is $\pi_{m+q}$. Note that this is the probability that the system is in state $m+q$ (all data subchannels are busy and $q$ users are in the distributed queue). We assume that the dropped users back-off and resend their requests in a way similar to new arrivals. Hence, the rate of customers leaving the system is $\lambda\left(1-\pi_{m+q}\right)$. The $\lambda \pi_{m+q}$ flow comes back to the system (cf. Fig. 2).

When a packet arrives (as a new packet or a retransmission), it is possible that the control subchannel is blocked by other nodes. For example, another node may be waiting for a CTS packet on the control subchannel. The RTS packet transmission for the newly-arrived data packet will have to be rescheduled. $^{4}$

The queueing system of the control subchannel is illustrated in Fig. 2. In a stable system, the new packet arrivals are always equal to the output rate of the control subchannel, $\lambda\left(1-\pi_{m+q}\right)$. Let $G_{a}$ represent the total aggregative traffic rate generated by a node. For each arrival in $G_{a}$, there is a chance, $p_{b}$, of seeing the channel busy (waiting for CTS packet) and the packet has to backoff immediately.

\footnotetext{
${ }^{4}$ It is possible that a packet will arrive to a busy node, which is sending another data packet or busy competing on the control channel. Such events are less likely when the number of active nodes are large.
} 
We now derive the relation between $p_{b}$ and $\lambda$. Based on the assumption that the traffic of successful RTS/CTS dialogues is a Poisson distribution with rate $\lambda$ and the fact that the arrival traffic is also Poisson, we can determine $p_{b}$ with the help from the Paradox of Residual Life [10] as follows. First we assume that the Poisson process with rate $G_{a}$ and the Poisson process with rate $\lambda$ are independent. Let random variable $X$ represent the time between consecutive successful RTS/CTS dialogue instants, i.e., the lifetime for a successful RTS/CTS dialogue. Note that $X$ is an exponentially distributed random variable with mean $1 / \lambda$. Let the arrival time of a data packet be $t$. Since the arrival is Poisson distributed, the probability density of the packet's arrival at any time instant for any given time interval is the same.

We need to determine the probability that it arrives within the last one time unit before an RTS/CTS dialogue succeeds. Let random variable $Y$ be the residual lifetime between any two successful RTS/CTS dialogues and its PDF $f_{Y}$ is given by $[10]$

$$
f_{Y}(y) d y=\operatorname{Pr}[y \leq Y \leq y+d y]=\frac{1}{\bar{X}}\left[\int_{y}^{\infty} f_{X}(x) d x\right] d y
$$

where $\bar{X}$ is the mean of $X(\bar{X}=1 / \lambda)$ and $f_{X}$ is the PDF of $X$. Therefore,

$$
f_{Y}(y)=\lambda e^{-\lambda y}
$$

The average rate of packets falling within the last one time unit before an RTS/CTS dialogue succeeds is given by

$$
G_{a} p_{b}=\int_{0}^{1} G_{a} f_{Y}(y) d y=G_{a}\left(1-e^{-\lambda}\right) .
$$

We have

$$
p_{b}=1-e^{-\lambda}
$$

Subsequently,

$$
G_{a}=\frac{G}{1-p_{b}}=G e^{\lambda} \quad \text { and } \quad G=G_{a} e^{-\lambda} .
$$

\section{Delay Analysis}

Let total data packet delay, $D$, be the duration between the instant when the packet is ready for transmission and the time when the transmission of the packet is completed. The total delay includes three components:

- $D_{1}$, the time for the request packet to reserve a data subchannel successfully;

- $D_{2}$, the time between the successful reservation and the end of packet transmission;

- $D_{3}$, the extra delay caused by the immediate reschedule of transmissions (the $G_{a} p_{b}$ branch in Fig. 2).

First we determine $E\left[D_{1}\right]$ as follows: Due to the chance of the queue being full (with probability $\pi_{m+q}$ ), a successful request sender may have to retransmit its RTS request. Let $N$ be the random number of successful requests sent by a user before it actually leaves the contention system (cf. Fig. 2). Let $Z_{i}, i=1,2, \ldots, N$, be the time duration for $i$-th successful
RTS/CTS contention. Thus, $D_{1}=\sum_{i=1}^{N} Z_{i}$. Since all $Z_{i}$ are i.i.d., the expected value of $D_{1}$ is

$$
E\left[D_{1}\right]=E\left[E\left[D_{1} \mid N\right]\right]=E[N E[Z]]=E[N] \cdot E[Z] .
$$

The second equality follows from the fact that

$$
E\left[D_{1} \mid N=n\right]=E\left[\sum_{i=1}^{n} Z_{i}\right]=n E[Z]
$$

so $E\left[D_{1} \mid N\right]=N E[Z]$. The random number $N$ has a geometric distribution with the successful probability $p=1-\pi_{m+q}$. We have $E[N]=1 /\left(1-\pi_{m+q}\right)$.

We derive $E[Z]$ as follows. Let $M$ be the random number of times of sending the request packet before it generates a successful RTS/CTS dialogue. $M$ has a geometric distribution with success probability $p=e^{-2 G}$ and mean $1 / p=e^{2 G}$. Assume that all nodes generate random backoff time based on an exponential distribution with mean $1 / \zeta$. Hence, all failed RTS requests last $2+Y^{(B)}$ units, where $Y^{(B)}$ is exponentially distributed with mean $1 / \zeta$. We calculate the value of $Z$ as the sum of these durations $Z=\sum_{i=1}^{M-1}\left(2+Y_{i}^{(B)}\right)+2$. Therefore,

$$
\begin{aligned}
& E[Z]=E[M-1] E\left[Y^{(B)}+2\right]+2 \\
= & \left(e^{2 G}-1\right)\left(\frac{1}{\zeta}+2\right)+2 .
\end{aligned}
$$

The expected value of $D_{2}$ can be obtained simply by applying Little's theorem to the queuing model discussed above. That is,

$$
E\left[D_{2}\right]=\frac{\sum_{n=0}^{m+q} n \pi_{n}}{\lambda\left(1-\pi_{m+q}\right)}
$$

We estimate the expected value of $D_{3}$ through the following method: When a packet arrives within the transmission duration of a CTS packet, its next few retransmissions may fall within the same CTS packet transmission duration. Let the transmission duration of a CTS packet be 1 and $f_{Y}(Y \leq y)$ be the probability that a packet arrives within the last $y$ transmission duration of a CTS packet, where $0 \leq y \leq$ 1. Note that there might be multiple arrivals within this $y$ period of time. Due to the memoryless property of exponential distribution in retransmission schedules, the average residual time delay of the next retransmission after the end of the CTS packet is still $1 / \zeta$. Hence, the expected waiting time of this packet is $E[T]=y+1 / \zeta$, where $y$ represents the delay caused by the blocking of the CTS packet transmission and $1 / \zeta$ is the expected arrival time of the next retransmission schedule. By calculating the average over all $y$, we have

$$
\begin{aligned}
E\left[D_{3}\right] & =\int_{0}^{1} f_{Y}(y)(1 / \zeta+y) d y \\
& =\int_{0}^{1}\left(y \lambda e^{-\lambda y}+\frac{\lambda}{\zeta} e^{-\lambda y}\right) d y \\
& =\frac{1}{\lambda}+\frac{1}{\zeta}-\left(1+\frac{1}{\lambda}+\frac{1}{\zeta}\right) e^{-\lambda} .
\end{aligned}
$$


Since the above calculations are in unit time, the overall delay can be calculated as $D_{\text {sec }}$

$$
\begin{aligned}
& E\left[D_{\text {sec }}\right]=L_{c} / R_{c} \cdot E[D] \\
= & L_{c} / R_{c} \cdot\left\{E\left[D_{1}\right]+E\left[D_{2}\right]+E\left[D_{3}\right]\right\} \\
= & \frac{(r+m) L_{c}}{r R}\left[\frac{\left(e^{2 G}-1\right)\left(2+\frac{1}{\zeta}\right)+2}{1-\pi_{m+q}}+\frac{\sum_{n=0}^{m+q} n \pi_{n}}{\lambda\left(1-\pi_{m+q}\right)}\right. \\
& \left.+\frac{1}{\lambda}+\frac{1}{\zeta}-\left(1+\frac{1}{\lambda}+\frac{1}{\zeta}\right) e^{-\lambda}\right]
\end{aligned}
$$

Note that both $G$ and $\zeta$ are in time units.

\section{E. Performance of the MAC-1 Scheme}

The performance of the MAC- 1 scheme can be calculated using a similar approach. We measure the overall delay of packet transmission, defined as the duration between the time when the data packet is ready and the time when successful data packet transmission ends. We denote the delay components of MAC- 1 scheme as $U_{1}, U_{2}$, and $U_{3}$ corresponding to $D_{1}, D_{2}$, and $D_{3}$, respectively (cf. Section II-D).

The calculation of $U_{1}$ is actually the $E[Z]$ given in (8). $U_{2}$ is the expected transmission time of a data packet:

$$
U_{2}=1 / \mu
$$

The calculation of $U_{3}$ should consider not only CTS packet transmission duration but also the duration of data packet transmission. Similar to the calculation of $E\left[U_{3}\right]$ (cf. (10)), we have

$$
\begin{aligned}
E\left[U_{3}\right] & =\int_{0}^{k+1}\left(y \lambda e^{-\lambda y}+\frac{\lambda}{\zeta} e^{-\lambda y}\right) d y \\
& =\frac{1}{\lambda}+\frac{1}{\zeta}-\left[(k+1)+\frac{1}{\lambda}+\frac{1}{\zeta}\right] e^{-(k+1) \lambda}
\end{aligned}
$$

where $k$ is the ratio of data packet size to the control packet size.

Since the above calculations are in the unit of $\gamma_{1}$, the overall delay can be calculated as $U_{s e c}$

$$
\begin{aligned}
& E\left[U_{\text {sec }}\right]=L_{c} / R \cdot E[U] \\
= & \frac{L_{c}}{R}\left\{\left(e^{2 G}-1\right)\left(2+\frac{1}{\zeta}\right)+2+\frac{1}{\mu}+\frac{1}{\lambda}+\frac{1}{\zeta}\right. \\
& \left.-\left[(k+1)+\frac{1}{\lambda}+\frac{1}{\zeta}\right] e^{-(k+1) \lambda}\right\} .
\end{aligned}
$$

\section{NumericAl AND Simulation Results}

The numerical and simulation results are based on an example network with 20 nodes. The overall channel data rate is 1 Mbps. The control packet length is $L_{c}=48$ bits and the data packet length $L_{d}=1024$ bits. For MAC- $m D$ schemes, the ratio between the control subchannel and the data subchannel, $r$, and the best average retransmission delay, $1 / \zeta$, are chosen as $r=0.45,0.72,1.69$ and $1 / \zeta=40,37,27$ [time-units] for $m=1,2,5$, respectively. We obtained these values through simulations. All packet delays are shown as traffic load increases to reach the system throughput, $S$ [2].

\section{A. Numerical Results}

We use (11) and (14) to calculate numerical results for different MAC- $m D$ and MAC-1 schemes. Their delay performance is shown in Fig. 3. It can be seen that the maximum achievable throughput of MAC- $m D$ increases with $m$. This is due to the higher flexibility to serve the successful RTS/CTS senders [2]. Interestingly, when the traffic load is low, the delay of MAC- $m D$ schemes with larger $m$ is higher than that of MAC- $m D$ schemes with smaller $m$. This is due to the overall transmission time of the control packets and the data packet. As $m$ increases, the transmission time of the control and data packets are generally longer. In Fig. 3, the delay of the MAC1 scheme is consistently lower than that of the MAC- $m D$ schemes.

Note that if we further increase the traffic load in our numerical calculation, the channel competition enters an unstable region. Therefore, we will see lower throughput $S$ and longer delay $U$ or $D$.

\section{B. Simulation Results}

In Fig. 4, we compare the simulation results with the numerical results that are based on (11) and (14). These results match quite well with each other when the throughput is lower. The discrepancy between numerical results and simulation results increases with throughput. The difference is larger for MAC- $m D$ schemes with smaller $m$ and the MAC-1 scheme. This might have been caused by our assumption of Poisson (re)transmission and infinite population in numerical calculations. Note that these assumptions are crucial for the derivation of total delay since all three delay components derived are strongly dependent to them.

In Fig. 5, the delay of MAC- $m D$ with variable packet length is shown to be longer than that of MAC- $m D$ with fixed packet length. The worse performance of networks with variable packet length could be caused by the mis-match between the control channel and the number of data subchannels. The MAC-1 scheme outperforms MAC- $m D$ schemes under both scenarios.

\section{Packet Transmission with Different Priorities}

We have also investigated packet delays when prioritized packets are involved. We assumed that there were two classes of priorities in our simulations: high priority and low priority. ${ }^{5}$ The portion of packets with high priority is either $50 \%$ or $20 \%$. The prioritized packets are processed as follows:

- High priority packets always preempt low priority packets in the local transmission queue of each node;

- When a high priority packet successfully reserves its channel usage, the channel reservation of a low priority packet by any node may be preempted. Therefore, extra cost may incur for this process.

The simulation results are shown in Fig. 6, in which we compare the delay performance of MAC- $m D(\mathrm{~m}=5)$ with

\footnotetext{
${ }^{5}$ We assume that the priorities of the packets are already in place, e.g. audio/video packets should have higher priority than regular data packets. The setting of packet priorities is out of the scope of this letter.
} 
the MAC-1 scheme. It can be seen that the packet delay of prioritized packets in MAC- $m D$ schemes is longer than that of the MAC-1 scheme. In the MAC- $m D$ scheme, as the ratio of prioritized traffic decreases, the packet delay of these prioritized traffic is lower. The delay performance of low priority packets are even worse in MAC- $m D$ schemes but similar in the MAC- 1 scheme.

\section{CONCLUSIONS}

Through analysis and simulations, we have investigated the delay performance of one class of multi-channel MAC schemes (the MAC- $m D$ schemes). In these schemes, one subchannel is used to transmit control packets such as the RTS and the CTS packets. Data packets are transmitted on the rest of the subchannels.

Based on our investigations, we have found that, in fullyconnected networks, the delay of the MAC- $m D$ schemes is usually higher than that of the MAC-1 scheme, which uses the single channel for control/data packet transmissions. The main reasons are the longer channel access delay and the longer packet transmission time. Prioritized traffic does not affect our conclusions.

Our investigation in this work is based on fully-connected networks with ALOHA contention resolution and error-free channel. We leave consideration of these issues in multihop networks and networks with CSMA/CD contention resolution, and the analysis of networks with prioritized traffic for future work.

\section{REFERENCES}

[1] S.-L. Wu, Y.-C. Tseng, C.-Y. Lin, and J.-P. Sheu, "A multi-channel MAC protocol with power control for multi-hop mobile ad hoc networks," Computer Journal, vol. 45, no. 1, pp. 101-110, 2002.

[2] Y. S. Han, J. Deng, and Z. J. Haas, "Analyzing multi-channel medium access control schemes with aloha reservation," IEEE Trans. on Wireless Communications, vol. 5, no. 8, pp. 2143-2152, August 2006.

[3] J. Deng, Y. S. Han, and Z. J. Haas, "Analyzing split channel medium access control schemes," IEEE Trans. on Wireless Communications, vol. 5, no. 5, pp. 967-971, May 2006.

[4] M. A. Marsan and F. Y. Neri, "A simulation study of delay in multichannel CSMA/CD protocols," IEEE Trans. on Communications, vol. 39, no. 11, pp. 1590-1603, November 1991.

[5] W. Yue and Y. Matsumoto, "Output and delay of multi-channel slotted ALOHA systems for integrated voice and data transmission," Telecommunication Systems, vol. 13, no. 2-4, pp. 147-165, June 2000.

[6] J. So and N. H. Vaidya, "Multi-channel MAC for ad hoc networks: Handling multi-channel hidden terminals using a single transceiver," in Proc. of ACM International Symposium on Mobile Ad Hoc Networking and Computing (MobiHoc '04), Roppongi Hills, Tokyo, Japan, May 24262004.

[7] C. Xu, G. Li, W. Cheng, and Z. Yang, "Multi-transceiver multiple access (MTMA) for mobile wireless ad hoc networks," in Proc. of IEEE International Conference on Communications (ICC '05), Seoul, Korea, May 16-20 2005, vol. 5, pp. 2932-6.

[8] Z. Tang and J. J. Garcia-Luna-Aceves, "Hop reservation multiple access for multichannel packet radio networks," Computer Communications, vol. 23 , no. 10 , pp. 877-886, 2000.

[9] W.-C. Hung, K. L. E. Law, and A. Leon-Garcia, "A dynamic multichannel MAC for Ad Hoc LAN," in Proc. 21st Biennial Symposium on Communications, June 2-5 2002, pp. 31-35, Kingston, Canada.

[10] S. K. Bose, An Introduction to Queueing Systems, Kluwer Academic/Plenum Publishers, New York, NY, 2002.

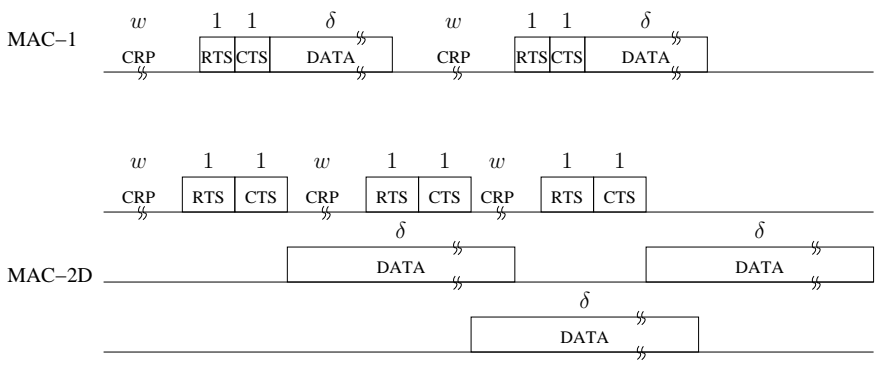

Fig. 1. Illustration of MAC-1 and MAC-2D. CRP represents Contention Resolution Period. In the MAC-1 scheme, all packets are sent on the single channel. In the MAC- $2 D$ scheme, control packets (RTS/CTS) are sent on the control subchannel. Two other subchannels are used to sent data packets. Note that the exact value of $\delta$ differs in each of the MAC schemes.

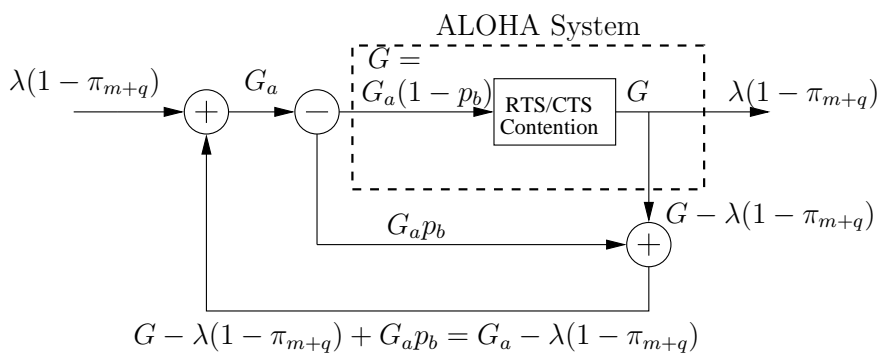

Fig. 2. Illustration of packet arrivals on the control channel.

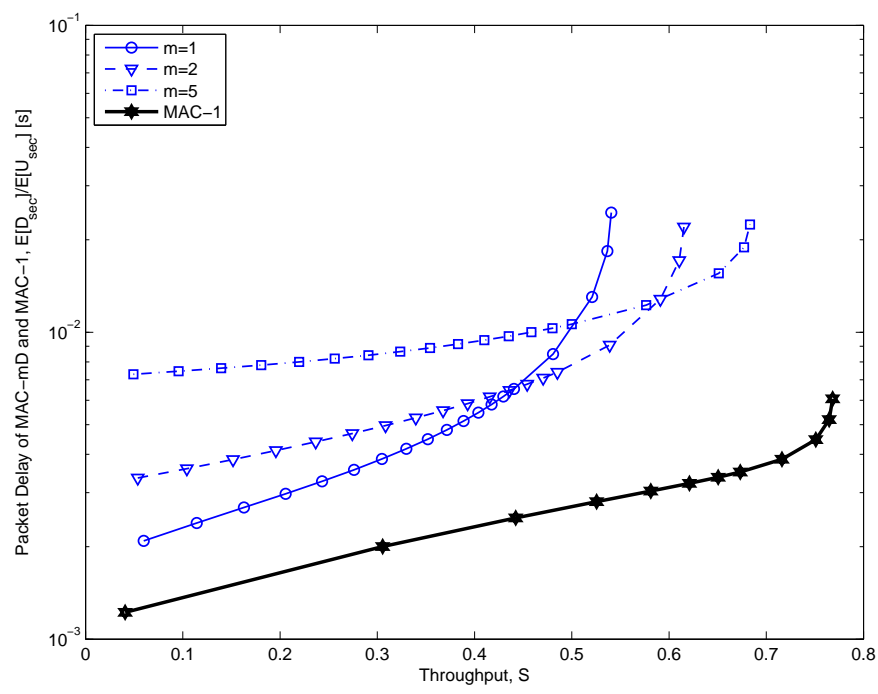

Fig. 3. Delay vs. Throughput for the MAC- $m D$ and the MAC- 1 schemes (numerical results based on (11) and (14)). 




Fig. 4. Comparison of numerical results and simulation results for the MAC$m D$ and the MAC- 1 schemes. Both the simulation and the numerical results assumed exponential packet length.

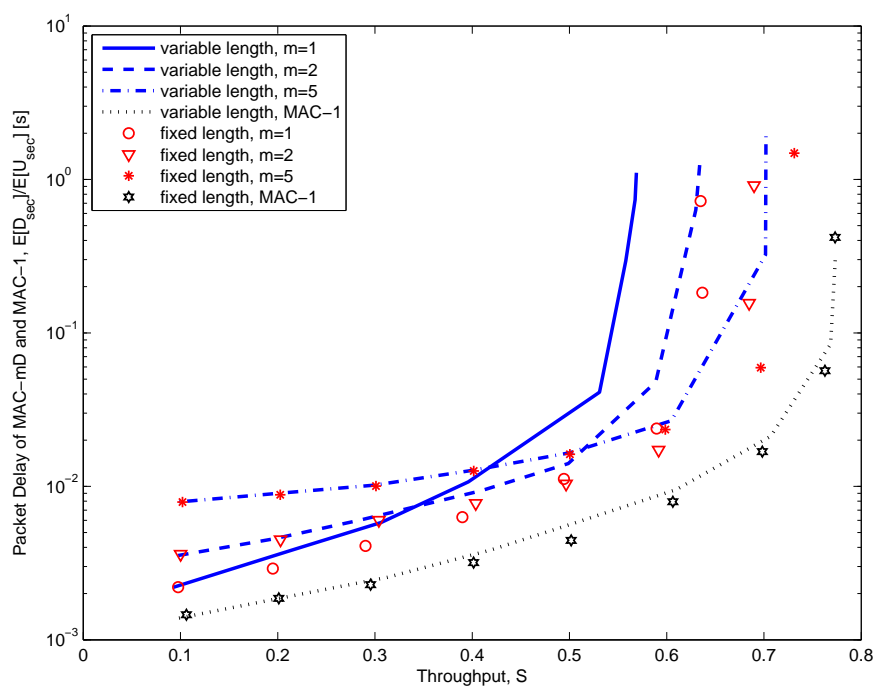

Fig. 5. Simulation results of delay performance for the MAC- $m D$ and MAC-1 schemes with variable packet length (exponential distribution) and fixed packet length.

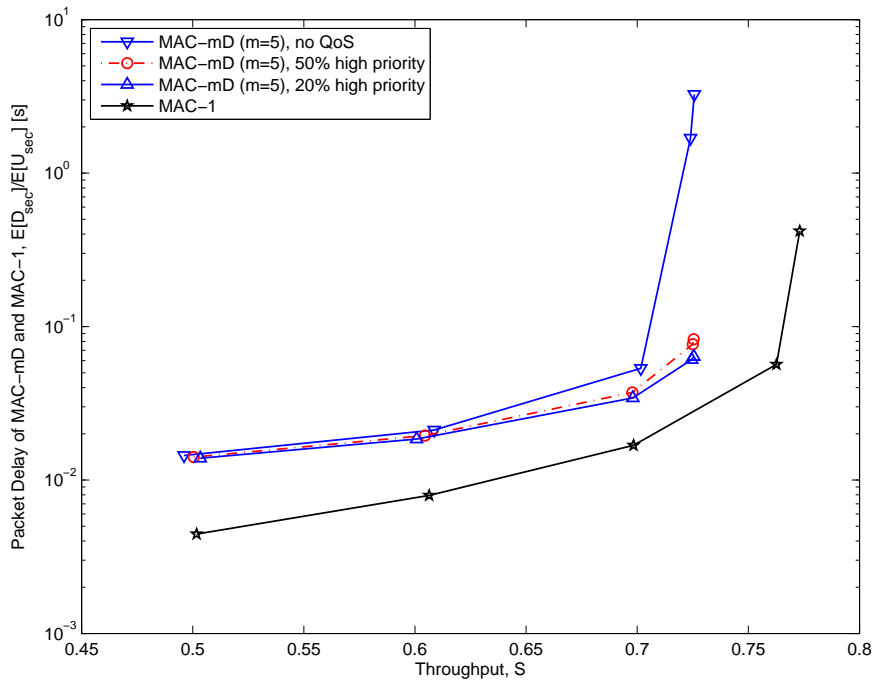

Fig. 6. Delay performance of prioritized traffic (simulation results). In the MAC- $m D$ scheme, the delay of high priority traffic is shown. Low priority traffic in the MAC- $m D$ schemes suffered longer delays. In the MAC-1 scheme, the delay of low priority traffic is very similar to that of high priority traffic. 